\title{
Hypersound excitation of magnetization and elastic displacement in case of magnetization reversal
}

\author{
Fanur Asadullin ${ }^{1,2, *}$, Dmitriy Pleshev ${ }^{1,2}$, Vladimir Vlasov ${ }^{2}$, Leonid Kotov ${ }^{2}$, Sergey Poleshchikov ${ }^{1}$, Vladimir Shavrov ${ }^{3}$, and \\ Vladimir Shcheglov ${ }^{3}$
}

${ }^{1}$ Saint-Petersburg state forest technical university named after S.M. Kirov, St-Petersburg, Russia

${ }^{2}$ Syktyvkar State University, Syktyvkar, Russia

${ }^{3}$ Institute of Radioengineering and Electronics of the Russian Academy of Sciences, Moscow, Russia

\begin{abstract}
The paper gives a description of hypersound magnetoelastic oscillations excitation along magnetisation reversal of normal magnetized single-layer and multi-layers ferrite plates, also provides definition of distinctive features of magnetic and elastic oscillations development. The relation of oscillations to the parameters of saturation magnetization, magnetic and elastic damping has been revealed.
\end{abstract}

\section{Introduction}

The magnetoelastic oscillations generation caused by magnetisation reversal is one of the interesting interaction mechanism of magnetic, elastic and magnetoelastic subsystems. This process can be executed in case of action on the plate by alternating magnetic field, hypersound oscillations as well as pulsed magnetisation reversal in a DC field. Dimensions and shape of a plate are fitted in such a way that avoid chance of uniform precession parametric decay into exchange spin wave [1].

\section{Geometry of the problem and basic equations}

The plane-parallel ferrite plate has thickness $d$. The external DC magnetic field $\mathbf{H}_{0}$ is applied perpendicularly to the plane of the plate as shown in Fig. 1. The problem is solved in a Cartesian coordinate system Oxyz. The plane Oxy of the coordinate system coincides with the plane of the plate. The coordinate axes are parallel to the edges of the cube crystallographic cell. The center of the coordinate system $\mathrm{O}$ is in the center of the plate, so that the plate planes coordinates are $z= \pm d / 2$. We consider only excitement of the shear elastic oscillations.

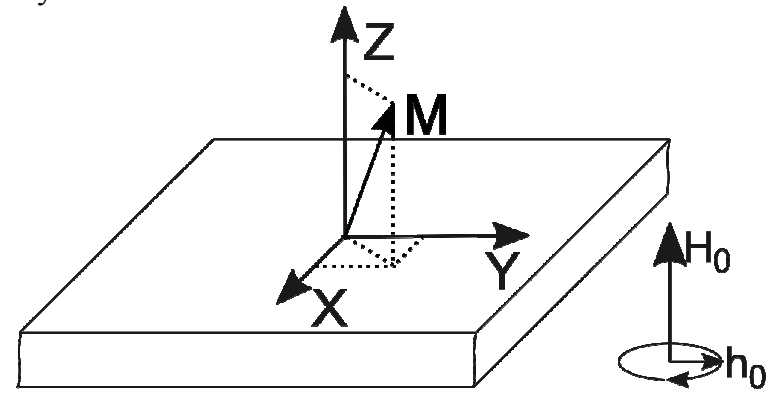

Fig. 1. Geometry of the problem.
Assuming that the total energy density of the plate $U$ in the field $\mathbf{H}=\left\{0 ; 0 ; \mathbf{H}_{0}\right\}$ equals the sum of magnetic, elastic and magnetoelastic energy densities, we obtain:

$$
\begin{aligned}
U= & -M_{0} H_{0} m_{z}+2 \pi M_{0}^{2} m_{z}^{2}+2 c_{44}\left(u_{x y}^{2}+u_{y z}^{2}+u_{z x}^{2}\right)+ \\
& +2 B_{2}\left(m_{x} m_{y} u_{x y}+m_{y} m_{z} u_{y z}+m_{z} m_{x} u_{z x}\right)
\end{aligned}
$$

where $\mathbf{m}=\mathbf{M} / M_{0}$ - normalized magnetization vector, $M_{0}$ the saturation magnetization of the magnetic plate, $u_{\mathrm{ij}}-$ the components of the strain tensor, $c_{44}$ - the elastic constant and $B_{2}$ - the magnetoelastic coupling constant.

The initial equations for the system are the LandauLifshitz-Gilbert equation and the equations for the elastic displacement vector components $\mathrm{u}_{\mathrm{x}, \mathrm{y}}$ :

$$
\begin{aligned}
& \frac{\partial \vec{m}}{\partial t}=-\gamma\left[\vec{m} \times \vec{H}_{e}\right]+\alpha\left[\vec{m} \times \frac{\partial \vec{m}}{\partial t}\right], \\
& \frac{\partial^{2} u_{x, y}}{\partial t^{2}}=-2 \beta \frac{\partial u_{x, y}}{\partial t}+\frac{c_{44}}{\rho} \cdot \frac{\partial^{2} u_{x, y}}{\partial z^{2}},
\end{aligned}
$$

where $\gamma$ - the gyromagnetic ratio; $\alpha, \beta$ - damping constants of the magnetic and elastic subsystems. The effective field is determined by the following expression:

$$
\overrightarrow{\mathbf{H}}_{e}=-\frac{1}{M_{0}} \cdot \frac{\partial U}{\partial \overrightarrow{\mathbf{m}}} \cdot
$$

The boundary conditions are:

$$
\left.c_{44} \frac{\partial u_{x, y}}{\partial z}\right|_{z= \pm d / 2}=-B_{2} m_{x, y} m_{z} .
$$

\footnotetext{
* Corresponding author: aff $@$ sfi.komi.com
} 
The system of equations [2] was solved numerically by the Runge-Kutta 7-8 orders method with control of the integration at every step length.

The material parameters used in the calculation are typical for Ni: $\mathbf{H}_{0}=10000$ Oe, $\rho=8.968 \mathrm{~g} \cdot \mathrm{cm}^{-3}$, $B_{2}=0.16 \cdot 10^{9} \mathrm{erg} \cdot \mathrm{cm}^{-3}, M_{0}=300 \div 600 \mathrm{G}, \quad c_{44}=1.2 \cdot 10^{11}$ $\mathrm{erg} \cdot \mathrm{cm}^{-3}, \alpha=0.035$.

\section{Development of magnetoelastic oscillations in a single-layer sample}

Assume that the plate is in the DC magnetic field $\mathbf{H}_{0}$. The $\mathbf{H}_{0}$ and $\mathbf{m}$ oriented in the negative direction of the axis $\mathrm{Oz}$ in the initial state. The DC field value is greater than the demagnetization field value, i. e. $\mathbf{H}_{0}<4 \pi M_{0}$. The $\mathbf{H}_{0}$ changes its direction from negative to positive at the initial time. Accordingly, constant and demagnetization fields become contra-directional. The system is in the unstable equilibrium and a minor fluctuation leads to the magnetization vector tends to rotate towards the field $\mathbf{H}_{0}$. As a result, the magnetization vector precesses around the axis $\mathrm{Oz}$ that accounts for hypersound oscillations excite due to magnetostriction.

The spatial evolution of magnetic oscillations and temporal development of elastic oscillations are shown in Fig. 2 and Fig. 3.
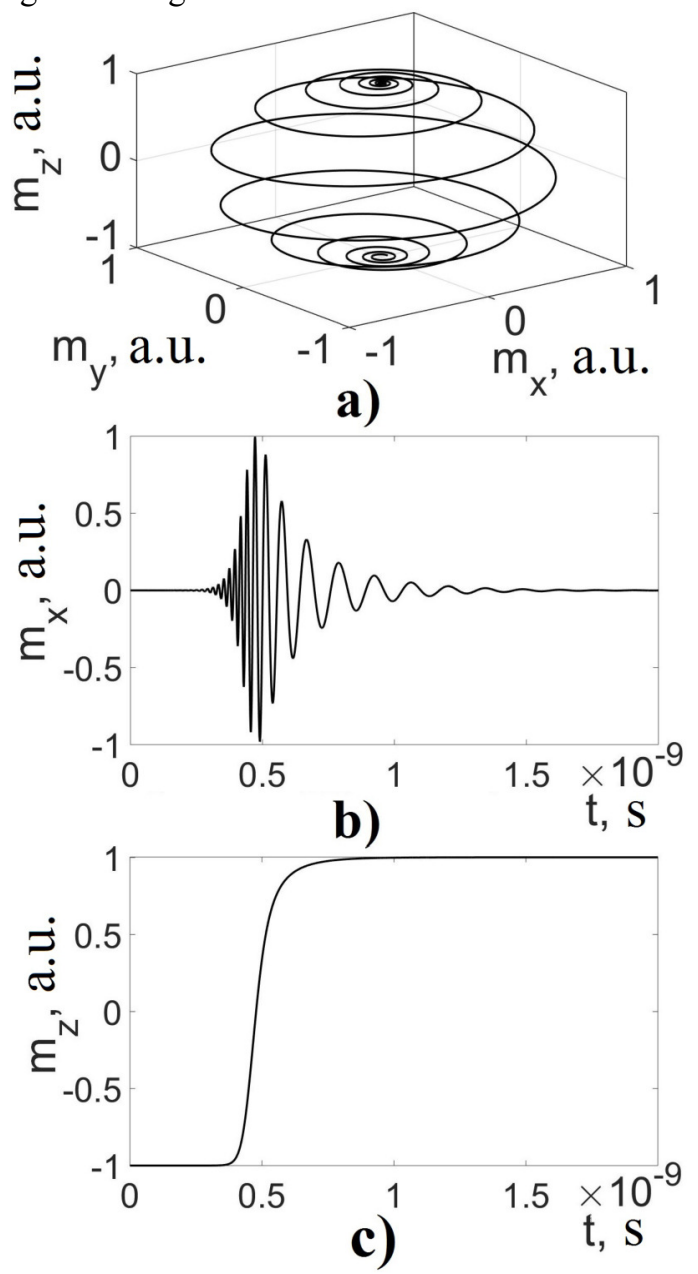

Fig. 2. a) development of magnetization components $m_{x}, m_{y}$, $\mathrm{m}_{\mathrm{z}} ; \mathrm{b}$ ) the $\mathrm{m}_{\mathrm{x}}$ time dependence; $\mathrm{c}$ ) the $\mathrm{m}_{\mathrm{z}}$ time dependence.
The travelling time of magnetization vector under Oxy-plane is different from the same over Oxy-plane. This is due to correlation of the DC field and the demagnetizing field directions, i.e. at the first stage, when the deviation of the magnetization vector from the field direction increases in time, the force that turns the magnetization vector to the field direction also increases, and at the second stage, when the deviation decreases, the force also decreases gradually to zero.

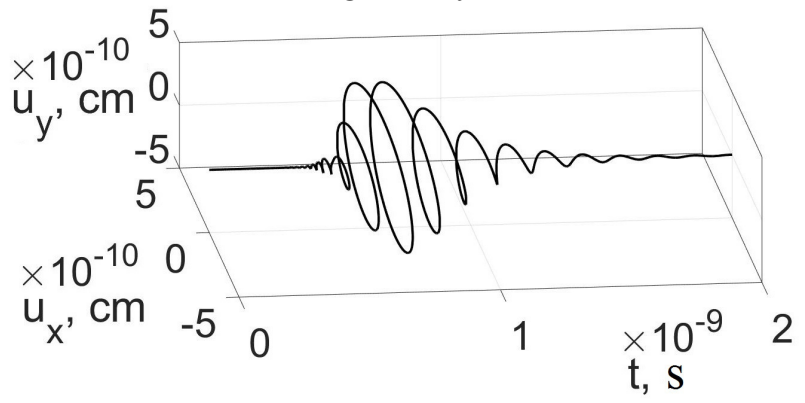

Fig. 3. The temporal development of elastic displacement components $\mathrm{u}_{\mathrm{x}}, \mathrm{u}_{\mathrm{y}}$.

Difference between magnetization precession frequencies is defined by the Kittel formula for normal magnetized infinite thin plate at the first and second stage.

Magnetization oscillations leads to elastic displacement oscillations due to magnetostriction. The elastic oscillations frequency is in accord with frequency of magnetization oscillations. This is apparent from spectra of elastic displacement and magnetization oscillations in Fig. 4. This is because magnetization oscillations relaxation time is much greater than elastic oscillation relaxation time and elastic oscillations follow magnetization oscillations in a quasi-stationary mode. The initiation of the minimum of amplitude of elastic oscillations is described in detail in the paper [3].
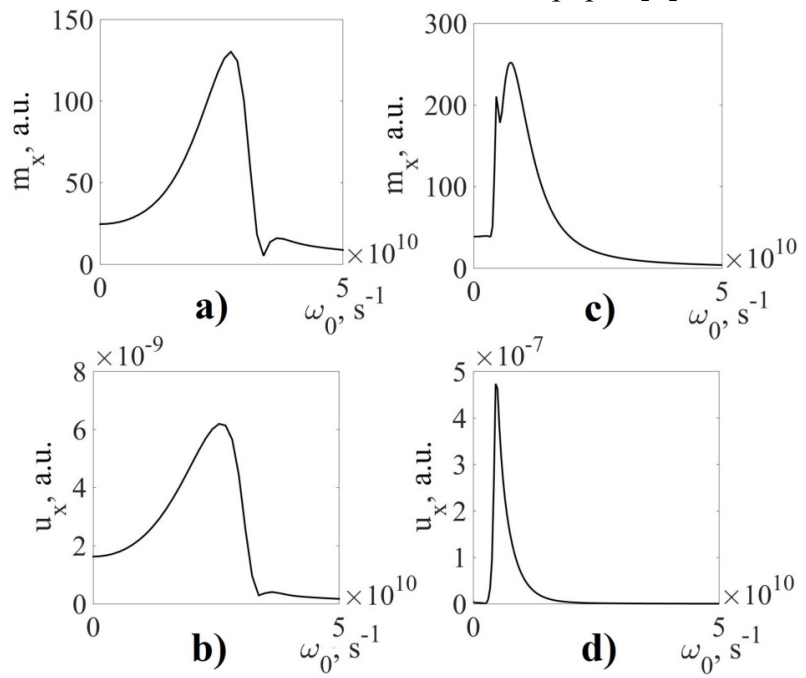

Fig. 4. Spectra of magnetic oscillations at the first stage (a) and at the second stage (c); spectra of elastic oscillations at the first stage (b) and at the second stage (d).

Fig.5 displays the dependencies of magnetization reversal process on the elastic damping parameter. It should be noticed here, that the time of maximum 
amplitudes development of elastic oscillations is kept in the range of elastic damping parameter values from $10^{3}$ to $10^{7} \mathrm{~s}^{-1}$ and from $10^{8}$ to $10^{11} \mathrm{~s}^{-1}$. However, a strong change of the maximum amplitudes development time at the second stage is observed between this intervals of elastic damping parameter.

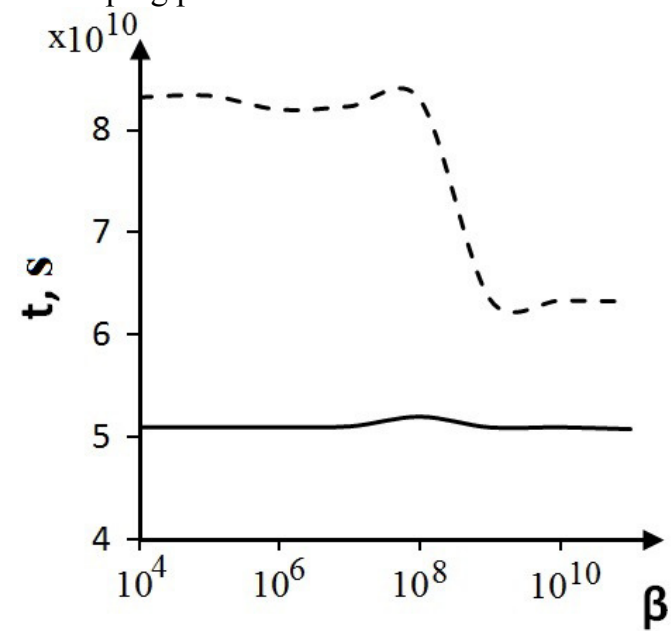

Fig. 5. The maximum amplitudes development time for $m_{x}$ (solid line) and $\mathrm{u}_{\mathrm{x}}$ at the second stage (dash line).

The constancy of the time of maximum amplitudes development stem from the following facts. In case of the small values of elastic damping parameter the elastic oscillation damping is defined by magnetic loss rather than elastic losses. And in case of the larger values of elastic damping parameter the elastic oscillations become nigh aperiodic. In addition to this, the elastic oscillation amplitude follows the magnetization amplitude.

The time of maximum amplitude development for elastic oscillations also depend on the saturation magnetization. The elastic oscillations at the second stage is most strongly influenced through the saturation magnetization. The fastest maximization of elastic oscillations amplitude is possible in the $330-560 \mathrm{G}$ range of the saturation magnetization (Fig. 6).

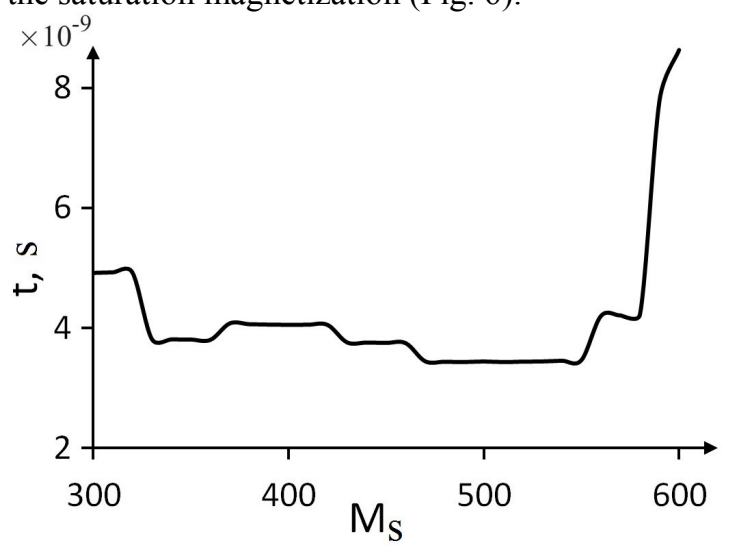

Fig. 6. Time of maximum amplitude development for elastic oscillations.

As is shown in Fig. 7 the amplitude of elastic oscillations exponentially decrease at the first stage of the magnetization reversal and exponentially increases together with increase of the saturation magnetization at the second stage.

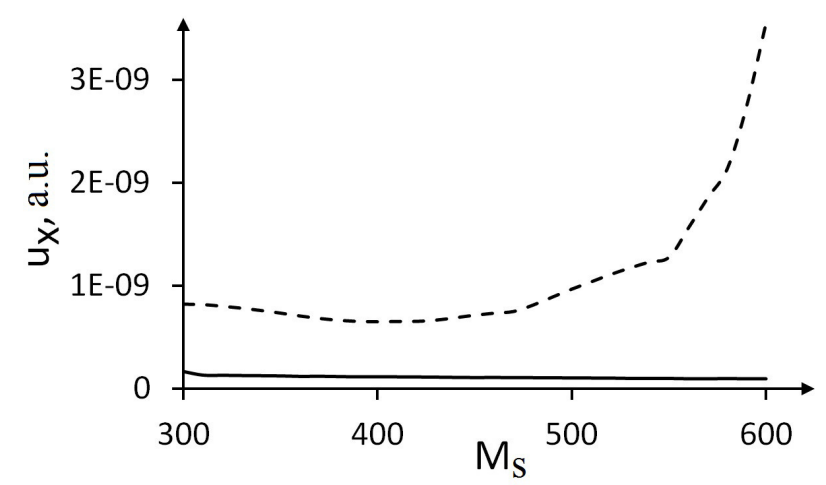

Fig. 7. Dependence of elastic oscillations maximum amplitude on the saturation magnetization.

\section{Development of magnetoelastic oscillations in a multilayer sample}

The development of magnetization oscillations and elastic displacements in multilayer plates with different layers material parameters of the sample corresponds to the development of oscillations in single-layer samples with equivalent parameters. This is due to weak interaction between layers in the absence of exchange interaction. There are the insignificant distortions of the elastic oscillations in the layers boundaries by this means.

\section{Conclusion}

Thus the dependencies of the maximum amplitudes development times of the magnetoelastic oscillations on the magnetic damping parameter and the saturation magnetization were defined. The values of the elastic damping parameter $\beta$ for the most efficient excitation of hypersound oscillations have been determined. The paper reveals the fact that the displacement amplitude of the excited elastic waves in the microwave range can exceed $10^{-9} \mathrm{~cm}$ using sample with $\mathrm{Ni}$ properties. It is equal to the level of strong nonlinear excitations and increases the level of linear excitation by more than orders of magnitude.

This work was supported by RFBR (grant no. 17-0201138-a, grant 17-57-150001 НЦНИ-а.).

\section{References}

1. V.S. Vlasov, L.N. Kotov, V.G. Shavrov, V.I. Shcheglov, Nonlinear excitation of hypersound in a ferrite plate under the ferromagnetic-resonance conditions, J. Comm. Tech. El. 54 (2009) 821-832.

2. M.S. Kirushev, V.S. Vlasov, D.A. Pleshev, F.F. Asadullin, L.N. Kotov, V.G. Shavrov, V.I. Shcheglov, Solid State Phenomena Vols 233-234 (2015) pp 73-78.

3. V.S. Vlasov, D.A. Pleshev, L.N. Kotov, V.G. Shavrov, V.I. Shcheglov, F.F. Asadullin, S.M. Poleshchikov, Solid State Phenomena Vols 233-234 (2015) pp 480-484. 